\title{
Efficient Compression and Querying of XML Repositories
}

\author{
Ramez Alkhatib \\ University of Konstanz \\ Box D 18878457 Konstanz, Germany \\ Ramez.Alkhatib@uni-konstanz.de
}

\author{
Marc H. Scholl \\ University of Konstanz \\ Box D 18878457 Konstanz, Germany \\ Marc.Scholl@uni-konstanz.de
}

\begin{abstract}
With the rapidly increasing popularity of XML as a data format, there is a large demand for efficient techniques in storing and querying XML documents. However XML is by nature verbose, due to repeatedly used tags that describe data. For this reason the storage requirements of $X M L$ can be excessive and lead to increased costs for data manipulation. Therefore, it seems natural to use compression techniques to increase the efficiency of storing and querying $X M L$ data. In this paper, we propose a new approach called SCQX for Storing, Compressing and Querying XML documents. This approach compresses the structure of an XML document based on exploiting repetitive consecutive tags in the structure, and then SCQX stores the compressed XML structure and the data separately in a robust storage structure that includes a set of access support structures to guarantee fast query performance. Moreover, SCQX supports querying of the compressed XML structure directly and efficiently without requiring decompression. An experimental evaluation on sets of XML data shows the effectiveness of our approach.
\end{abstract}

\section{Introduction}

With the increasing popularity of XML [11] as a data representation format, the problem of efficiently processing and storing XML documents poses interesting challenges for database research. In recent years, various methods to store and retrieve the XML data have been developed. Many relational databases are adding XML support to their systems [7], but typically, native XML databases will be more efficient since they store the XML data persistently in its native form, avoiding a costly transformation into relations and vice versa. Several XML query languages have been proposed, but XPath and XQuery [11] are currently the most commonly used languages to query XML. All these languages are based on path expressions to navigate the nested structure of XML. Therefore, the efficient support of path expressions over XML data plays a major role in the field of XML. A variety of ways is available to provide robust storage and efficient query processing. These ways fall into two categories: a) those which focus on decreasing the cost of XML storage $[2,1]$. While the representation of XML documents has excessive information redundancy, reducing the size of the structure of the XML tree in a compact representation enhances query processing speed; b) those, which are based on numbering schemes $[4,9]$ to identify the relationship between XML elements. This identification plays a crucial role in efficient XML query processing. In order to combine the advantages of both techniques we developed a new approach for Storing, Compressing and Querying XML documents (SCQX). Specifically, in this paper we are concerned with providing, first, an effective algorithm for the compression of the structure of XML documents based on exploiting repetitive consecutive tags in the structure of the XML documents; by using a labeling scheme that has a small storage requirement maintains the relationships among XML tags after compression. Second, a complete and robust storage structure for compressed XML, including a set of access support structures to guarantee a fast query performance, is presented. In summary, we make the following contributions:

- SCQX provides the guarantee that data values will only be accessed on demand.

- SCQX processes queries directly over the compressed structure without full or partial decompression.

- We implemented and evaluated SCQX over a number of test data sets. The results show the effectiveness of our approach.

The rest of this paper is organized as follows: In Section 2, we discuss related work. Section 3 introduces the SCQX approach. Section 4 presents a performance evaluation. Finally, Section 5 concludes the paper and discusses future work. 


\section{Related Work}

In recent years, several path indexing, labeling and numbering schemes have been proposed for query processing of XML documents. The basic idea of these schemes and methods is that each node is assigned a unique (integer) number in such a way that it takes constant time to determine the relationship of any two nodes. We also use a numbering scheme in our compressed XML representation that maintains all the relationships among XML nodes after the compression. The technique of vertical partitioning developed for relational databases divides a table into multiple tables defined over subsets of the attributes. This partitioning lets queries typically scan less data and thus increases query performance. XMill [5] used this strategy in the XML context, it separates the structural information of an XML document from the content and compresses them separately, but it focused on compressing XML and not directly addressed querying of the compressed document. Buneman et al. [1] extend the skeleton compression technique [2] to facilitate the processing of XQuery based on the vectorization approach which is an extreme form of vertical partitioning. In this approach, main memory data structures are used for the compressed skeleton, while external memory data structures hold text contents. However, this technique has some drawbacks: First, sometimes the compressed skeleton is still too large, so that it does not fit into main memory. Second, compressed skeleton will always be scanned in its entirety to identify the relevant data vectors. In contrast, SCQX stores the compressed structural information with its labeling information in Structure table which can be stored in an external memory. The result is a storage Structure that is immune to the main memory limitations of skeletal processing, while at the same time providing additional access methods. The MonetDB/XQuery [8] implementation is based on the XPath Accelerator [4] that is one of the most comprehensive proposals for schema-oblivious mappings. It effectively used region encoding techniques for query evaluation. MonetDB and its Pathfinder extensions provide remarkably good performance; therefore the comparison to it is very interesting. Section 4 reports on performance comparisons between SCQX and MonetDB.

\section{SCQX Approach}

In this section, we present the principles behind our approach for representing and compressing the structure of XML trees. Then we shall describe the storage model of SCQX and discuss how XPath queries are processed within the compressed format.

\subsection{The Level-Order Numbering Scheme}

The main purpose of the numbering scheme is to allow a quick identification of structural relationships between nodes. To achieve this aim, every node in the document tree is labeled with a unique identifier. The ID is chosen in such a way, that we can compute the relationship between two nodes in the same document from the ID alone. In our approach, we use the level-order numbering scheme. In this scheme, a unique integer $i d_{U}\left(u_{i}\right)$ is assigned to each element node $u_{i}$ while traversing the document tree in levelorder (breadth-first traversal). Figure 1 displays an XML document with level-order ids. In addition, we store with each node the parentID (not shown in Figure 1). Also, for each text node ${ }^{1} t u_{i}$ we store the ID of its parent element node $u_{i}$. We use this encoding scheme because it has important properties: first, it is easy to infer relationships between parent and child or sibling nodes. Second, it has fixed size. Finally, it allows a faithful representation of the XML document after the compression.

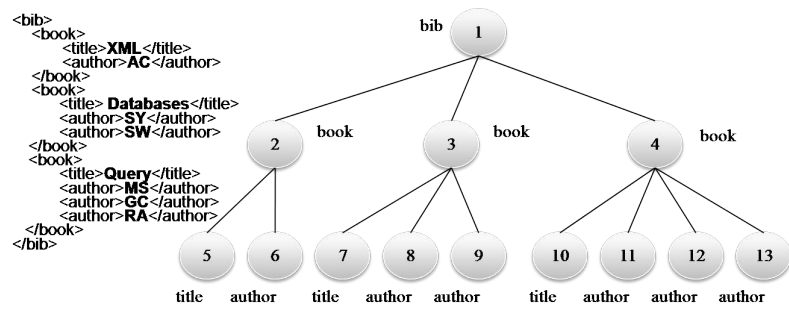

Figure 1. XML document structure with levelorder ids (shown inside nodes)

\subsection{Compression Principles}

In many cases, the volume of large XML documents mainly comes from their text contents. In contrast, most database queries tend to focus on structural aspects with only occasional access to character contents. Therefore compressing the structure separately from the data is helpful to accelerate access to the data and to make the structure occupy as little storage as possible [5].

To compress an XML structure with SCQX, the input XML is first scanned using, e.g., a SAX parser. The XML tags are then compressed using our algorithm which basically exploits the repetition of similar sibling nodes of XML structure, where "similar" means: elements with the same tag name. A cardinality counter is assigned to the first node of such similar sibling nodes, reflecting the number of repetitions. The other similar sibling nodes will not be stored in

\footnotetext{
${ }^{1}$ For simplicity's sake, attribute nodes are disregarded in this paper, they can be treated like text nodes.
} 
our storage model. Each node in the compressed structure represents a set of nodes in the uncompressed tree. Note that our algorithm works while parsing the tree without extra costs, and it works even if the consecutive branches of trees do not exactly have the same substructure. We should also remark an interesting property of this technique: it does not rely on a DTD or XML schema.

Returning to Figure 1, consider the three book nodes. These three nodes are similar. Therefore, we can replace them by the first node, which has ID value 2 and parentID 1, and assign 3 to this node's cardinality counter; and so forth for all other nodes of the given XML structure. Figure 2 displays the compressed structure of the XML document of Figure 1 , where the crossed-out nodes will not be stored.

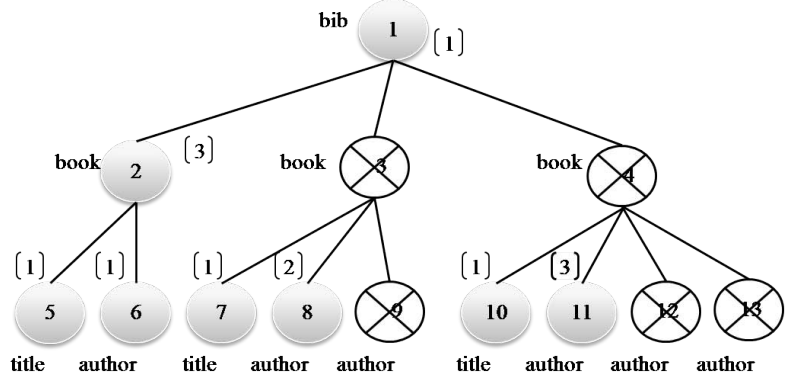

\section{Figure 2. Compressed structure with unique numbers of elements and cardinality coun- ters (in parentheses).}

\subsection{The Storage Model}

The storage of XML documents plays an important role, because efficient query processing is critically dependent on the chosen storage structure [3]. In this section, we describe our proposed storage model for representing XML documents, which stores the data values and the compressed structural information separately. We assign to each XML element node $u_{i}$ of the structure T a unique integer $i d_{U}\left(u_{i}\right)$ obtained by level-order numbering (Section 3.1). Let $\mathrm{P}$ be the set of all paths in an XML structure. Each path $\rho \in P$ is a sequence $i d_{U}\left(u_{0}\right), i d_{U}\left(u_{1}\right), \ldots, i d_{U}\left(u_{\tau p(\rho)-1}\right)$, where $\tau p(\rho)$ is the length of the path. Node $u_{0}$ is always the root node of the XML structure. SCQX is built on the data structures that are listed below and guarantees a compact mapping of XML files:

Structure table stores, for each node $u_{i}$ of the compressed structure, the tag name with its own ID $\left(i d_{U}\left(u_{i}\right)\right)$, the ID of its parent, and its cardinality counter.

Value table stores each text contents with its id (TID) and the ID of its element parent.
The tags as well as the text contents are indexed in separate hash structures; the index entries are referenced by integer values. The internal representations, shown on the right in Figure 3, store all information as integers. Both the parent and the cardinality counters are stored in an integer array, and the ID and TID value are implicitly given by the array position. The node tables for the XML document in Figures 1 and 2 are shown in Figure 3.

Path index: For better query performance, we create a path

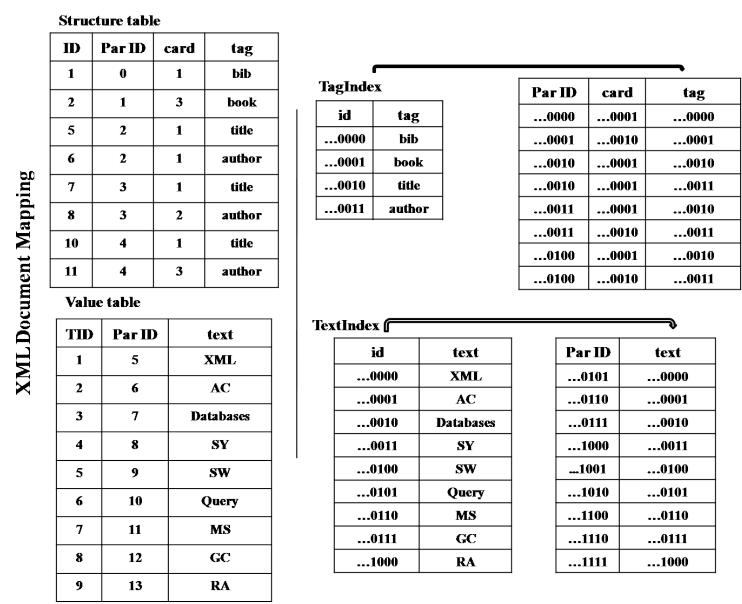

Figure 3. Relational XML mapping (left), internal table representation in SCQX (right)

index, which contains a unique integer (so-called pathID) $i d_{P}\left(\rho_{i}\right)$ for each path $\rho_{i} \in P$. The pathID is the same for multiple element nodes with the same path. Thus, element nodes (ID = 5) and (ID = 7) refer to two different TITLE nodes, but the paths leading to these nodes are both expressed as /bib/book/title. As such, they have the same pathID value 3 (see table 1). This index can be implemented by a hash structure. In addition, the index is extended by references to the structure table's ID values, resulting in an inverted list.

\section{Table 1. Path table for the example XML doc- ument}

\begin{tabular}{|l|l|}
\hline pathID & Path Expr \\
\hline 1 & /bib \\
2 & /bib/book \\
3 & /bib/book/title \\
4 & /bib/book/author \\
\hline
\end{tabular}

Hash index: This index is an implementation of a linked list hash structure. In order to reduce the storage requirements and to access the stored nodes (tags or text contents 
or paths) quickly, the hash index is flattened into integer arrays, which are sized by a power of two, and instead of using the conventional modulo in which the array size is based on the calculation of prime numbers, we used the bitwise AND operator to cut down the calculated hash value to the array size. This approach is fast, and the index can be quickly resized and rehashed during index creation.

\subsection{Query Evaluation}

In order to evaluate basic XPath queries, (with all XPath axes) node tests and basic text predicates (with textual, numeric, and positional matches), an XPath parser has been implemented. We have chosen different algorithms for queries with or without positional predicates and we performed some optimization steps for simplifying and reformulating the XPath query.

A full path or simple queries such "/" or "//" can be easily answered by performing some matching on the Path index. For example, to answer a full path such as Q1: /BIB/BOOK/SECTION/TITLE, it requires exact match for this path in the path index and if it is found, return its path ID and a list of the structure table's ID values referenced by this pathID.

For a path expression containing the //-axis, such as Q2: //SECTION/TITLE, answering Q2 is similar to Q1, except that it requires suffix match for SECTION/TITLE in the path index, again we yield a set of pathIDs and set of lists of the structure table's ID values referenced by these pathIDs. The situation is similar for path expressions containing a wildcard or //-axis in the middle of the path expression, such as Q3: /BOOK/*/TITLE or Q4:/BOOK/SECTION//TITLE. In the latter case, the exact match for /BOOK/SECTION and suffix match for TITLE yield a set of path IDs.

For path expressions containing predicates, such as Q5: /BOOK/SECTION[TITLE = "DBMSs"], that fit the pattern "path=value", first we find all the ID values referenced by the pathID of /BOOK/SECTION/TITLE. After that, we check which one of the resulted ID values has a text value equal to "DBMSs" using exact matching on the value table, yielding a set of ID values. The structure table is then used to return the parentID to obtain the SECTION in the result. Note that the ancestor and ancestor-or-self axes are also supported using prefix matching; the structure table also permits to efficiently evaluate child-parent, preceding-sibling, and following-sibling relationships. Inferring the other relationships also uses some matching and /or seek on tables.

To accelerate the predicate queries, the existing text value index is extended by references to the structure table's ID values, resulting in an inverted list. We apply this index when we find exact string comparisons in the query; the input XPath query is rewritten to call the text content index. Descendant steps are converted to ancestor steps and vice versa. It is also very effective when nested loops with predicate joins are encountered, reducing the quadratic to a linear execution time without the need for additional algorithms. For example Q5:/BOOK//SECTION[text $($ )="DBMSs"]/TITLE. "DBMSs" is matched against the text content index. The resulting set (ID values) is matched against the SECTION self and the BOOK ancestor and then TITLE child.

\section{Experimental Results}

For the evaluation, we have examined the comparative performance of SCQX on three XML documents which cover a variety of sizes, application domains, and document characteristics. The XML documents used in our experiment are listed below:

Shakespeare: a set of the plays of William Shakespeare marked up in XML for electronic publication with 7.9 MB size.

XMark: an XML file from the XMark Benchmark [10], the files with the sizes of $11 \mathrm{MB}$ and $112 \mathrm{MB}$ are used.

SWISS-PROT: is a protein sequence database with 110 MB size.

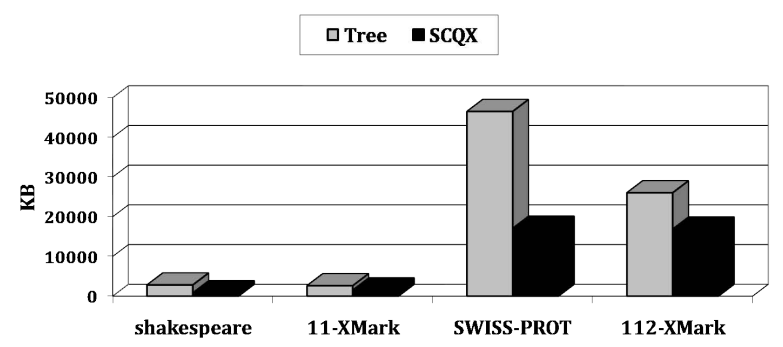

\section{Figure 4. Comparison of storage require- ments (size in kB)}

\subsection{Size of Structure}

The memory requirements that are needed to store the tree structure of the XML document are measured according to the size of its data model representation. The typical data model presents an XML document as a tree structure in which each node has a name pointer, three pointers to its parent, its first child, and the next sibling. This means to represent the structure of an XML document as a tree, each node in this tree needs at last 16 bytes [6]. In contrast to that, only a name pointer, parent pointer, and cardinality counter are needed to store each node of the compressed 


\section{Table 2. The elapsed time (in milliseconds) and the XPath queries used in the performance comparison of SCQX with MonetDB}

\begin{tabular}{|c|c|c|c|c|}
\hline $\mathbf{N r}$ & Queries & MonetDB & SCQX & Hits \\
\hline \multicolumn{5}{|c|}{ Dataset : SwissProt } \\
\hline QP1 & //Entry//Descr & 1502,18 & 52,34 & 344814 \\
\hline QP2 & //Entry/Keyword[text 0 = "Rhizomelic chondrodysplasia punctata"] & 1920,45 & 28,45 & \\
\hline \multirow{2}{*}{ QP3 } & //Entry/Org $[$ text $0=$ "Piroplasmida"] & 1764,18 & 22,72 & 13 \\
\hline & \multicolumn{4}{|l|}{ Dataset : Shakespeare } \\
\hline QSI & /SHAKESPEARE/A_AND_C/PLAY/ACT/SCENE/SPEECH/SPEAKER & 1111,63 & 11,27 & 1179 \\
\hline QS2 & //PLAY/ACT[4] & 857,18 & 6,27 & 37 \\
\hline QS3 & //PLAY/SCENE//STAGEDIR & 657,81 & 48,36 & 6224 \\
\hline QS4 & $/ /$ SPEECH/SPEAKER $[\operatorname{text}(0=$ "PHILO" $]$ & 1533,76 & 7,18 & 2 \\
\hline \multirow[t]{2}{*}{ QS5 } & //PLAY/ACT/SCENE/SPEECH/SPEAKER[text0-'Steward'] & 1345,54 & 14,54 & 6 \\
\hline & \multicolumn{4}{|l|}{ Dataset : XMark } \\
\hline QX1 & /site/regions/africa/item/description/parlist/listitem & 1225,65 & 21,72 & 57 \\
\hline QX2 & /site/regions/namerica/item/name/text 0 & 1322,15 & 4,21 & 1000 \\
\hline QX3 & //regions//item & 1023,3 & 6,76 & 2175 \\
\hline QX4 & /site/regions//item/description & 1323,73 & 3,45 & 2175 \\
\hline QX5 & $/$ site/people/person/profile/education[text $0=$ "College"] & 1531,31 & 14,73 & 182 \\
\hline QX6 & //africa//item[location $=$ 'United States $]$ /name & 1704,42 & 87,56 & 47 \\
\hline QX7 & /site/regions/asia/item[shipping]/description & 1371,94 & 134,56 & 200 \\
\hline QX8 & /site/open auctions/open auction/bidder $[1]$ increase/text 0 & 1812,83 & 604,64 & 1080 \\
\hline
\end{tabular}

structure in our model (see Section 3.3). Figure 4 shows the storage requirements of SCQX in comparison with the tree structure representation. Note that the SCQX leads to more compact representation.

\subsection{Test Queries}

We compare the performance of SCQX with MonetDB/XQuery 4.20.0 [8], a full-fledged XQuery and XPath query engine, whereas SCQX is currently limited to XPath 1.0 queries. Therefore, we chose a subset of the query set of the benchmark $(\mathrm{QX})$ that is compatible with the XPath supported by our system. We also used a set of queries (QS) for the Shakespeare dataset and (QP) for the Protein dataset. The full list of queries is summarized in table 2 . We ran all queries on a Pentium $4 \mathrm{PC}$ at $3 \mathrm{GHz}$ with $1 \mathrm{~GB}$ RAM, running Windows XP. SCQX is implemented in Java. Each query was run 10 times; the average of those 10 runs is reported. The execution time includes the time for compiling and processing a query. The results of the experiments are presented in table 2 , where the elapsed query processing time is measured in milliseconds. It is shown in the experiments that the SCQX response time for almost all queries is spectacularly fast. Especially when the indexes are applied to the query; they guarantee query times less than 30 milliseconds, even for a large document such as Swiss-protein dataset.

\section{Conclusions}

In this paper, we propose SCQX, an approach that supports efficient query processing on compressed XML structures. The main idea of SCQX is to take advantage of a particular, level-order XML numbering scheme and compression techniques to support query processing. First experimental results show that our approach improves query performance and produces a smaller, compact XML structure, without losing any information. As future work we will concentrate on modifying our approach to facilitate updates of the compressed XML documents.

\section{References}

[1] P. Buneman, B. Choi, W. Fan, R. Hutchison, R. Mann, and $\mathrm{S}$. Viglas. Vectorizing and querying large $\mathrm{xml}$ repositories. In ICDE, pages 261-272, 2005.

[2] P. Buneman, M. Grohe, and C. Koch. Path queries on compressed xml. In $V L D B$, pages 141-152, 2003.

[3] D. Florescu and D. Kossmann. Storing and querying xml data using an rdmbs. IEEE Data Eng. Bull., 22(3):27-34, 1999.

[4] T. Grust. Accelerating xpath location steps. In SIGMOD Conference, pages 109-120, 2002.

[5] H. Liefke and D. Suciu. Xmill: An efficient compressor for xml data. In SIGMOD Conference, pages 153-164, 2000.

[6] D. Megginson. Imperfect XML: Rants, Raves, Tips, and Tricks ... from an Insider. Addison-Wesley Professional, 2004.

[7] M. Nicola and B. V. der Linden. Native $\mathrm{xml}$ support in $\mathrm{db} 2$ universal database. In VLDB, pages 1164-1174, 2005.

[8] U. of Amsterdam. Monetdb. http://sourceforge.net/projects/monetdb/.

[9] P. E. O’Neil, E. J. O'Neil, S. Pal, I. Cseri, G. Schaller, and $\mathrm{N}$. Westbury. Ordpaths: Insert-friendly xml node labels. In SIGMOD Conference, pages 903-908, 2004.

[10] A. Schmidt, F. Waas, M. L. Kersten, M. J. Carey, I. Manolescu, and R. Busse. Xmark: A benchmark for xml data management. In $V L D B$, pages 974-985, 2002.

[11] W3C. XML, XPath, XQuery. http://www.w3.org. 